\title{
On the common topological conditions for shear-coupled twin boundary migration in bcc and hcp metals
}

\author{
A. Serra ${ }^{\dagger}$, N. A. Kvashin, N. Anento \\ †a.serra@upc.edu \\ Department of Civil \& Environmental Engineering, Universitat Politècnica de Catalunya, BarcelonaTech, \\ Jordi Girona 1-3, Barcelona, 08034, Spain
}

\begin{abstract}
The most abundant deformation twins in metals with hcp and bcc crystallographic structures, i. e. (10-12) and (112) respectively, proceed with the same mechanism of deformation under an applied shear stress, i.e. shear-coupled twin boundary migration. Despite the differences in the atomic structure and degrees of symmetry of bcc and hcp crystals, the topological aspects that rule the processes of growth and shrinkage of these deformation twins are equivalent. In this paper, we revisit the atomic level processes, occurring at the interfaces, through which both coherent twin boundaries displace in a conservative manner and accommodate deformation. These processes include the creation of a twin boundary dislocation that acts as a source of disconnections, namely, line defects at the interface that have both dislocation and step character. The glide of disconnections along the twin boundary is responsible for the displacement of the interface, implying the growth or shrink of the twin. We describe the interaction of these twins with crystal dislocations and show that the product of the interaction is a source of disconnections. We show that these interactions can be described in terms of the structure of the interfaces and the Burgers vector of dislocations. The study is based on the topological theory of interfacial defects and we show the usefulness of the dichromatic pattern associated to each interface in the prediction and analysis of the interactions.
\end{abstract}

Keywords: twin boundary, twin growth, topological aspects, shear-coupled migration, molecular dynamics.

\section{Introduction}

For an atomic level process to occur at a grain boundary (GB), it is necessary that both, the topology (geometry) and the interatomic interactions (physics) accomplish certain constrains. The former refers to the atomic structure of both the GB and the interacting defects. The latter refers to the atomic interactions, which are material dependent and affect the energy balance, the local stresses needed to trigger reactions, Peierls stress to move GB dislocations and the effect of temperature.

The theory of interfacial defects [1-4] covers the first aspect bringing the necessary background to analyze the intrinsic defects associated to the GB. Since the atomic structure of each GB depends on the crystal structure, in the literature there are descriptions of individual GBs, at most, grouped by the crystal structures [5-14], but few overviews on the properties common to GB of different crystal structures are reported [15]. Twin boundaries (TB) are a particular case of GBs. TBs have been extensively studied for hcp and fcc metals [16-20] but in less extend for bcc metals [22-24]. Twins are extended defects that form as a deformation mode complementary to slip modes for the accommodation of plastic deformation [25]. In hcp crystals, because of their low symmetry, twinning is a prevalent deformation mechanism with, at least, seven twinning modes involving different twinning planes, although not all of them appearing in all hcp metals. The most abundant and common twin for all hcp metals is the $\{10-12\}$ twin, despite the fact that it has high formation energy compared to other twins of the same metal, proving that the interface energy is not a dominant parameter for the nucleation and growth of twins. The main twin mode in bcc metals is the $\{112\}$ twin. The coherent $\{112\}$ interface has the lowest energy among the $\langle 110\rangle$ tilt grain boundaries because the atoms at the interface present the perfect coordination number [26]. Thus, these two twins are the most abundant and they appear in all metals with hcp and bcc crystal structure respectively. In fact, although they belong to two different crystal structures with different symmetries, the atomic level processes related to the conservative displacement of their boundaries under a shearcoupled TB migration, are ruled by equivalent mechanisms.

In this paper, we revisit the common features of the coherent TBs and, based on their crystallography, we explain their high mobility and relevant interaction with crystal dislocations. The paper begins with the definition of the main concepts and tools necessary for the description of the processes at the $\mathrm{TB}$, followed by the interactions with dislocations for each TB pointing out their similarities.

\section{Methodology}

\subsection{Visualizing TB line defects: the dichromatic pattern}

According to Pond's theory of interfacial defects $[1,2]$, the set of Burgers vectors $(\mathrm{Bv})$ of the admissible $\mathrm{TB}$ dislocations is the difference of translation vectors of the 
two lattices $\left(\boldsymbol{b}_{n / m}=\boldsymbol{t}_{\lambda}-\boldsymbol{t}_{\mu}\right)$. An easy tool to visualize these $\mathrm{Bv}$ is the dichromatic pattern (DP) [27] shown in Fig. 1. The DP is the superposition of lattice sites of the two crystals [white/yellow $(\lambda)$ and black $(\mu)$ ] in their tilt related orientations, with the sites of the twinning plane of the two crystals in coincidence. Thus, any lattice site of the coincident plane may be taken as the origin of both $\boldsymbol{t}_{\lambda}$ and $\boldsymbol{t}_{\mu}$, i.e., the Bv's are represented by arrows from black to yellow sites in Fig. 1a and from black to white sites in Fig. 1b.

The TB dislocations that step the $\mathrm{TB}$ are named disconnections. The height of the step associated to a disconnection is $h=\boldsymbol{n} \cdot\left(\boldsymbol{t}_{\lambda}+\boldsymbol{t}_{\mathrm{u}}\right) / 2$ where $\boldsymbol{n}$ is normal to the TB plane. The position of the $\mathrm{Bv}$ in the $\mathrm{DP}$ indicates the height of the step and the stepping up or down of the TB. We adopt the notation $\boldsymbol{b}_{n / m}$ to refer to all dislocations (crystal and TB dislocations), being $n$ and $m$ the number of TB planes of $\lambda$ and $\mu$ along $\boldsymbol{t}_{\lambda}$ and $\boldsymbol{t}_{\mu}$, respectively.

Among disconnections, we name 'twinning disconnections' (TD) the glissile ones, with $\mathrm{Bv}$ parallel to the $\mathrm{TB}$, responsible for the growth and shrinkage of the twin. In this paper, $\boldsymbol{b}_{1 / 1}$ (Figs. 1a and $2 \mathrm{a}$ ) is a TD of the (112) twin and $\boldsymbol{b}_{-2 /-2}$ (Fig. $1 \mathrm{~b}$ and $2 \mathrm{~b}$ ) is a TD of the (10-12) twin.

The other TB dislocations in Fig. 1 are the result of the interaction of the twin with a crystal dislocation, as detailed below.

\section{2. $M D$ simulations}

The results presented were obtained by molecular dynamic (MD) simulation $[12,13,16,28-30]$. The computational system consisted of two half-crystals (white upper $(\lambda)$ and black lower $(\mu)$ ) in twin orientation separated by either a planar twin boundary or a boundary containing a TD in edge orientation with line direction parallel to the $x$ (tilt) axis. This bi-crystal model had periodic boundary conditions in the interfacial glide plane $x-y$ of the twinning disconnection, and fixed outer boundaries in the form of rigid blocks of atoms in the $\pm z$ directions $[31,32]$. With this construction, a TD moving in the $\pm y$ direction crosses one periodic boundary and re-enters at the opposite side of the simulation box. Due to the step character of the disconnection, the twin interface has been displaced in the $z$ direction by the step height
( $\pm 2 d$ for the $\{10-12\}$ and $\pm d$ for the $\{112\}$; $d-$ interplanar distance). Crystal dislocations were introduced on the upper side of the twin boundary.

\section{Twinning disconnections for the bcc-(112) and hcp-(10-12) twins}

TDs are interfacial defects; therefore, they can only glide along the TB. The motion of TDs is the essential mechanism for deformation twinning $[30,33]$. The passage of a TD along the $\mathrm{TB}$ produces a relative displacement of the twin and matrix by the Burgers vector, $b_{n / n}$, and the TB concomitantly advances by $h=n d$; the shear accommodated is $s=b / h$.

The Bv of the TD for the $(1 \overline{1} 2)$ twin is $\vec{b}_{1 / 1}=1 / 6[1 \overline{1} \overline{1}]$; $\left|b_{1 / 1}\right|=\sqrt{3} / 6 \cdot a_{0}=0.288 a_{0}$ ( $a_{0}$ is the lattice parameter). The resolved shear stress of this disconnection in $\alpha$-Fe is about $20 \mathrm{MPa}$ [34] compared to $82 \mathrm{MPa}$ for the $1 / 2<111>\{110\}$ edge dislocation [35]. Since it steps the TB by one plane, no shuffles are required during its glide [30] to restore the lattice of the transformed crystal and the disconnection is highly mobile.

The $\mathrm{Bv}$ of the TD for the $(10 \overline{1} 2)$ twin is $\vec{b}_{-2 /-2}=\alpha[10 \overline{1} \overline{1}]$; where $\alpha$ is approximately 0.0645 for the lattice parameter ratio $c / a=1.5952$ (see [36] for details), $\left|b_{-2 /-2}\right|=0.152 a_{0}$. The resolved shear stress of this disconnection in $\mathrm{Zr}$ is about 2.5 $\mathrm{MPa}$ compared to $5.5 \mathrm{MPa}$ for the $<\mathrm{a}>$ crystal dislocation [37]. It steps the TB by two planes and shuffles are required to restore the lattice (see [30] for details). The properties of this disconnection, i. e., small Bv (see Fig. 1b), wide core (see Fig. 2b) and easy shuffles [30], cause its low Peierls stress and high mobility.

The shear-coupled TB migration for the (10-12) twin, due to the glide of TDs, has long been studied by theory and simulation. Recently, Molodov et al [38] proved experimentally the shear-coupled migration in $\mathrm{Mg}$ and confirmed the value of the twinning shear $(s=0.126)$ in excellent agreement with the theoretical value $s=\gamma^{2}-3 /(\gamma \sqrt{3})$; where $\gamma$ is the $c / a$ ratio.

Thus, the growth and shrinkage under stress of these twins depends on the existence of TDs that can be created, either directly as dipoles at the pristine TB or by defects at the TB acting as sources of disconnections. For a source to be efficient, it should be linked to the TB and move together with it. In section 5, one such source of disconnections is described for each twin.

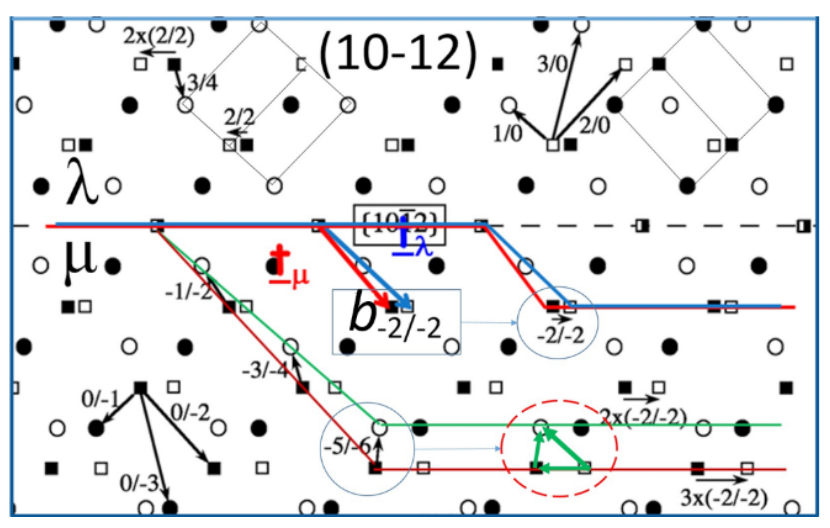

b

Fig. 1. (Color online) Projection of the dichromatic patterns along the tilt axis. Red and blue arrows are translation vectors of the $\lambda$ and $\mu$ crystals. (112) TB in bcc crystal. Black arrows are TB dislocations: $b_{1 / 1}$ is the twinning disconnection; $b_{1 / 1}$ is a TB dislocation (a). (10-12) TB in hcp Zr. $b_{1 / 0}$ is $1 / 3<1-210>$ Burgers vector; $b_{-2 /-2}$ is a twinning disconnection and $b_{-5 /-6}$ is a disconnection (b). The outline of two unit cells in each dichromatic pattern are shown. 


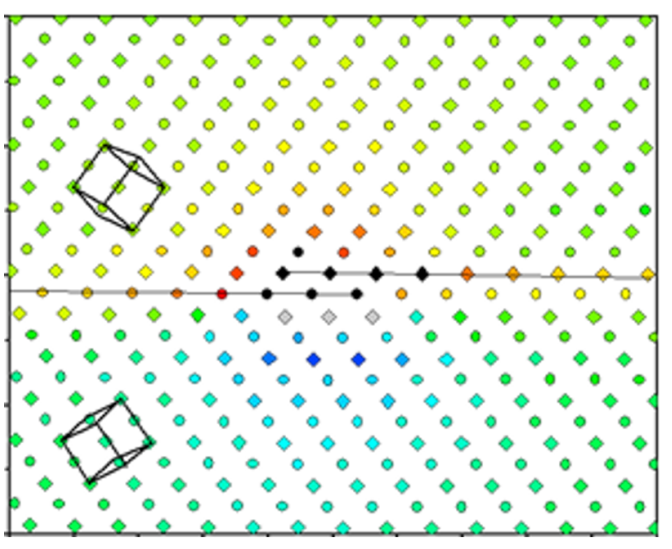

a

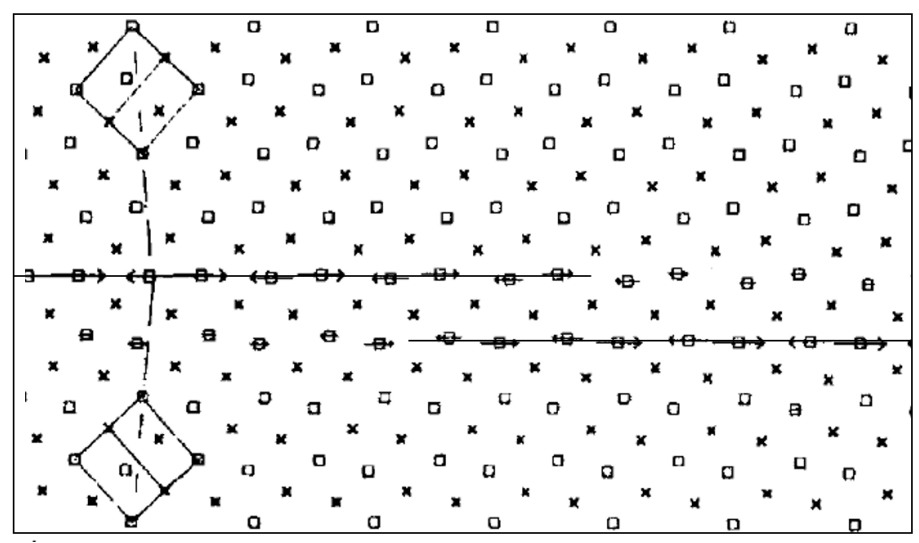

b

Fig. 2. (Color online) Projection along the tilt axis of the twin boundaries showing TD. $b_{1 / 1}$ at the (112) twin (a). $b_{-2 /-2}$ at the (10-12) twin (b).

\section{Shear-coupled TB migration: Creation of TDs at the TB}

The displacement of the TB under an applied stress by glide of TDs occurs if the topology of the boundary is appropriate for the creation of TDs. For this to occur, the Bv and step height of the disconnection should be small such that the energy and stress needed to create the dipole are reasonable and no other shear mode is more effective.

When this necessary condition is accomplished, the dipole can be created under high shear stress at the pristine boundary or, under lesser stress, by a source of TDs, as described below. The position (right or left) of each disconnection of the dipole is the appropriate to accommodate the stress, i.e., to run in the correct direction. Therefore, for a given sense of stress, the sense of motion of the TB depends on its topology, since a disconnection with positive Bv may step the boundary up or down as a function of the topology of the TB. An example is given in Fig. 3 where we have assumed that the positive $\mathrm{Bv}$ steps down the TB, as in the two twins presented here.

\section{Interaction of the twin boundary with a crystal dislocation: source of disconnections}

The following processes are common to both TBs:

- TD dipoles are created under shear stress. These TDs are highly mobile.

- A crystal dislocation interacting with the TB is fully absorbed and the reaction depends on the sign of the $\mathrm{Bv}$ of the incident dislocation.

- A TB dislocation acts as a source of TDs. The mechanism allows a conservative displacement of the TB.

In the following, the interactions are described for each twin and for each orientation of the crystal dislocation.

\subsection{Interaction of $1 / 2<111>$ dislocation with the} (11) twin boundary

\subsubsection{Edge dislocation $b_{2 / 0}=1 / 2[1 \overline{1} 1]$}

The edge dislocation, denoted $b_{2 / 0}$ in the DP of Fig. 1a, is absorbed by the (112) TB and transforms as: $\boldsymbol{b}_{2 / 0}=\boldsymbol{b}_{1 /-1}+\boldsymbol{b}_{1 / 1}[29]$.

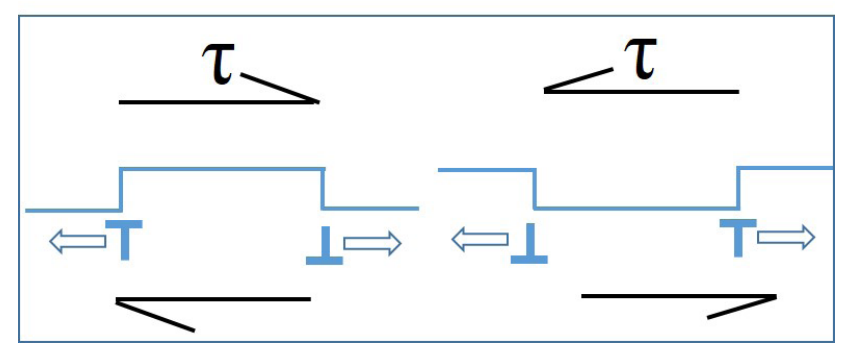

Fig. 3. Disconnection dipoles under shear stress $(\tau)$. White arrows show the motion of disconnections. Left figure: the shear displaces the TB upwards. Right figure: the shear displaces the TB downwards.

The decomposition of $\boldsymbol{b}_{2 / 0}$ is indicated in the green circle at the DP of Fig. 1a, together with the translation vectors of $\lambda$ and $\mu$ crystals (broken symmetries) associated to $\boldsymbol{b}_{1 /-1}=1 / 3[1 \overline{1} 2]_{\lambda}$. The DP shows the lines along the $\lambda$ and $\mu$ crystals indicating the cutting and joining procedure (in a Volterra sense) associated to $\boldsymbol{b}_{1 /-1}$ defect that does not step the TB. Once the reaction takes place the TD $\boldsymbol{b}_{1 / 1}=1 / 6[1 \overline{1} \overline{1}]_{\lambda}$ glides away.

Under a positive shear stress, $\boldsymbol{b}_{1 /-1}$ is a stress concentrator that favours the creation of $\boldsymbol{b}_{ \pm 1 / \pm 1}$ dipoles. The following reaction occurs: $\boldsymbol{b}_{1 /-1}+\left(\boldsymbol{b}_{1 / 1}+\boldsymbol{b}_{-1 /-1}\right)=\boldsymbol{b}_{2 / 0}+\boldsymbol{b}_{-1 /-1}$. While the $\boldsymbol{b}_{-1 /-1}$ runs away the $\boldsymbol{b}_{2 / 0}$ is located a plane above and the whole TB has moved by the height of the step of the TD and the process is repeated. The creation of disconnection dipoles, and hence the coupled shear-GB motion, is sustained once the threshold stress is reached (about $2 \mathrm{GPa}$ for Fe). Thus the $\boldsymbol{b}_{1 /-1}$ defect is a source of TDs that moves together with the TB in a conservative manner, i. e. no diffusion of atoms is needed. The process is reversible by reversing the sign of the stress.

\subsubsection{Edge dislocation $b_{-2 / 0}=1 / 2[\overline{1} 1 \overline{1}]$}

There is repulsion between this dislocation and the TB. If a negative shear stress is applied forcing the dislocation to approach the $\mathrm{TB}$, a concentration of stress is produced in the region between both defects that induces the creation of a disconnection dipole that, by gliding apart, moves the TB down. The dislocation is not absorbed, even it does not reach the $\mathrm{TB}$, but it facilitates the conservative displacement of the TB downwards. The threshold shear stress for a sustained 
motion is about $1.6 \mathrm{GPa}$ in $\mathrm{Fe}$. In this case, reversing the stress would move the dislocation apart from the TB and the process would end.

\subsubsection{Mixed dislocation $b_{1 / 0}=1 / 2[111]$}

This case shows three possible reactions in the DP; the MD simulation of the reaction confirmed the one occurring. The screw part of the $\mathrm{Bv}\left(\sqrt{2} / 2 a_{0}\right.$ along the tilt axis) is common to $\lambda$ and $\mu$ crystals and the edge part $\left(1 / 2 a_{0}\right)$ has a component perpendicular to the $\mathrm{TB}$ and a component parallel that has magnitude $\left|\boldsymbol{b}_{1 / 1}\right|$.

Thus, the dislocation could be transmitted from $\lambda$ to $\mu$ if two $\boldsymbol{b}_{1 / 1}$ disconnections could be added transforming a translation vector of $\lambda$ crystal into a translation vector of $\mu$ crystal. This reaction is not energetically favourable and does not occur even at a temperature of $T=600 \mathrm{~K}$. Thus, there is no transmission of the dislocation through the TB. There is another possible reaction energetically favourable described in the DP: $\boldsymbol{b}_{1 / 0}=\boldsymbol{b}_{-1 /-2}+\boldsymbol{b}_{2 / 2}$. This reaction does not occur either because both disconnections are sessile and they cannot go apart. Thus, the $\boldsymbol{b}_{1 / 0}$ is attached to the TB but it is not absorbed by the TB. When the TB is displaced by a shear-coupled migration, it drags the dislocation. See [29] for details.

\subsection{Interaction of $\langle a\rangle$ basal dislocation with the

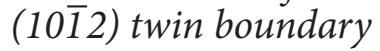

\subsection{1. $b_{1 / 0}=1 / 3[11 \overline{2} 0]$}

The basal dislocation $\vec{b}=1 / 3[11 \overline{2} 0]$, denoted as $b_{1 / 0}$ in the DP (Fig. 1b) is inclined at $60^{\circ}$ to the interface. It is spontaneously absorbed by the (10 $\overline{1} 2) \mathrm{TB}$ and transformed as: $\boldsymbol{b}_{1 / 0}=3 \boldsymbol{b}_{2 / 2}+\boldsymbol{b}_{-5 /-6}[16,39]$. The decomposition of $\boldsymbol{b}_{1 / 0}$ is indicated in the red circle of the DP as sum of green vectors. The three TDs, stepping up the TB by $h=6 d$, glide away; the disconnection $\boldsymbol{b}_{-5 /-6}$ steps the TB down (Fig. 4 a) introducing a facet in the TB. In the DP of Fig. $1 b$ there are the lines indicating the cutting and joining procedure associated to the $\boldsymbol{b}_{-5 /-6}$ defect. These lines show the facet that is formed by joining the basal plane of the $\lambda$ crystal and the prism plane of the $\mu$ crystal. The length of this facet can increase by adding $\boldsymbol{b}_{2 / 2}$ disconnections creating an incommensurate $\mathrm{TB}$, reported in the literature as basal/prismatic boundary $(\mathrm{B} / \mathrm{P})[40,41]$. The energy of this interface is comparable to the energy of the (10-12) TB. The boundary $\mathrm{B} / \mathrm{P}$ has been recognized experimentally [42] and it is crucial, for instance, in the development of the twin embryo $[43,44]$.

If a shear stress is applied, dipoles of TDs $\boldsymbol{b}_{2 / 2}$ are generated by the stress-concentrating effect of the immobile defect's core. Continued application of the shear stress leads to repeated production of TD dipoles with concomitant migration of the TB a distance $2 d$ for every pair created and lateral displacement of the $\boldsymbol{b}_{-5 /-6}$ defect [45], as shown in Fig. $4 \mathrm{~b}$. Thus, the source of disconnections follows the TB in its displacement. This process is conservative because the climb of the $\boldsymbol{b}_{-5 /-6}$ disconnection is compensated by the expansion of the TD dipole outwards from the defect. The situation is equivalent to a single TD moving through the facet from left to right because the climb down of $\boldsymbol{b}_{2 / 2}$ is compensated by the climb towards the right of $\boldsymbol{b}_{-5 /-6}[45]$.

The displacement of the TB with the facet created by the $\boldsymbol{b}_{-5 /-6}$ disconnection is reversible. If the sense of the shear stress is reversed, the sense of the displacement is reversed.

\subsection{2. $b_{-1 / 0}=1 / 3[\overline{1} \overline{1} 20]$}

If the sign of the basal dislocation is changed, the reaction at the TB produces a facet prismatic/basal $(\mathrm{P} / \mathrm{B})$ (contrary to $\left.b_{1 / 0}\right)$, i. e., the riser of the TB defect is not coincident with the glide plane of $\boldsymbol{b}_{-1 / 0}$, as shown in Fig. 5. The reaction at the TB depends on which partial dislocation $\left(30^{\circ}\right.$ or $\left.90^{\circ}\right)$ is getting first at the interface but it is controlled by the strong attraction of the $90^{\circ}$ partial. The reactions are as follows: $90^{\circ}$ partial leading $\boldsymbol{b}_{-1 / 0}=2 \boldsymbol{b}_{-2 /-2}+\boldsymbol{b}^{30}+\left(\boldsymbol{b}^{90}+\boldsymbol{b}_{4 / 4}\right)$. Then, the $90^{\circ}$ partial alone is absorbed and the $30^{\circ}$ partial is left off the TB separated by a stacking fault. The $30^{\circ}$ partial alone would be repelled by the TB but if it is leading, then it is pushed by the attraction of the $90^{\circ}$ and the whole dislocation is transformed into a TB disconnection with emission of two TDs: $\boldsymbol{b}_{-1 / 0}=2 \boldsymbol{b}_{-2 /-2}+\boldsymbol{b}_{3 / 4}$.

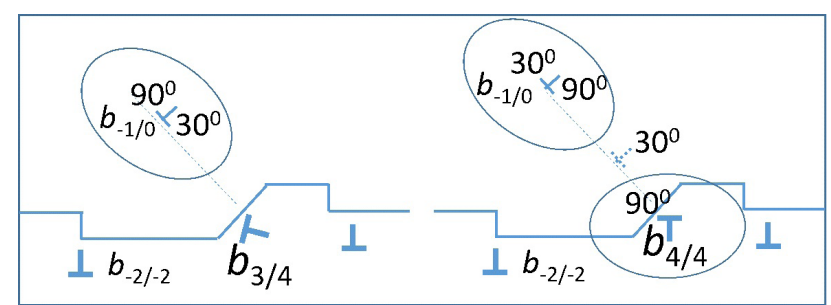

Fig. 5. Absorption of $\boldsymbol{b}_{-1 / 0}$ a crystal dislocation by the (10-12) TB and creation of the TB defect. Left) the $\boldsymbol{b}_{-1 / 0}$ glides on a basal plane with the $30^{\circ}$ partial leading. Right) the $\boldsymbol{b}_{-1 / 0}$ glides on a basal plane with the $90^{\circ}$ partial leading. See text for details.

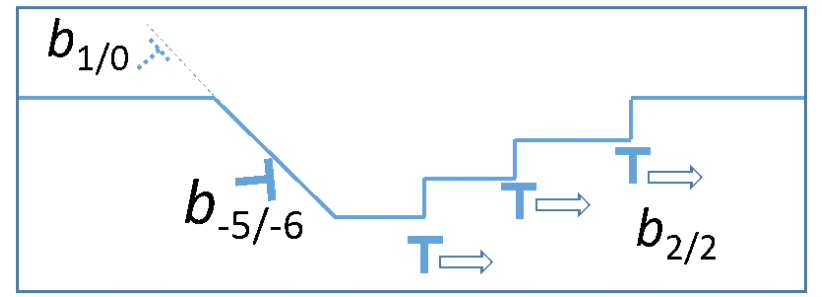

a

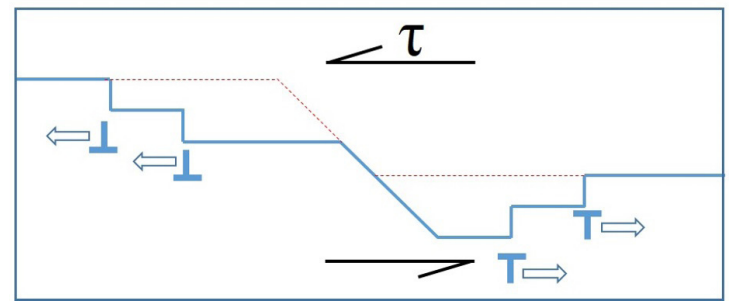

b

Fig. 4. Absorption of a $\boldsymbol{b}_{1 / 0}$ crystal dislocation by the (10-12) TB and creation of the $b_{-5 /-6}$ defect (a). Displacement downwards of the TB under the twinning stress $\tau$ (b). The $b_{-5 /-6}$ defect in the (1012) twin boundary acting as source of TDs. When a stress is applied new $b_{2 / 2}$ TD loops expand outwards and allow the boundary to migrate a distance $2 d$ for each one created. 
When a shear stress is applied to TB containing either $\boldsymbol{b}_{3 / 4}$ or $\boldsymbol{b}^{90}+\boldsymbol{b}_{4 / 4}$, they tend to move in the opposite sense as expected for the $\boldsymbol{b}_{-1 / 0}$. This is because the direction of motion of the TB depends only on the topology of the twin boundary, as explained in the previous section. Thus, a negative stress would move the TB down and a positive stress would move the TB up. The $\boldsymbol{b}^{90}+\boldsymbol{b}_{4 / 4}$ defect follows the TB by moving along the glide plane of the $\boldsymbol{b}_{-1 / 0}$. This can be understood as if the former $\boldsymbol{b}_{-1 / 0}$ is dragged by the TB with a riser formed by $\boldsymbol{b}_{4 / 4}\left(=2 \boldsymbol{b}_{2 / 2}\right.$ in two different planes). The same reasoning cannot be applied to the $\boldsymbol{b}_{3 / 4}$ defect that follows the TB for a negative shear but undergoes a reversal of the decomposition for a positive shear [45].

\section{Summary and conclusions}

We have described and compared the atomic level processes that occur at two twin boundaries of different crystallographic structures (bcc and hcp), when they exhibit shear-coupled twin boundary migration.

The events, enumerated below, lead to the conservative motion of the TBs under a shear stress. They are common to the $\{112\}$ TB in bcc and $\{10-12\}$ TB in hcp crystals and would explain the existence of these twins in all metals of the corresponding crystallographic structure. These processes are not exclusive of these twins; they may exist in other TBs or GBs that accomplish the following topological requirements:

- The shear-coupled migration is accommodated by the glide of TDs that are created at the TB either as dipole pairs in the pristine interface or by a source of disconnections. These TDs are highly mobile.

- The interaction of a crystal dislocation with the TB produces a TB dislocation that acts as a source of TDs.

- The crystal dislocation is absorbed by the $\mathrm{TB}$ and transformed into TB dislocations by a reaction that follows the conservation of the Burgers vector.

- The Burgers vector of the source of disconnections undergo a conservative climb due to its interaction with the TDs that glide along the TB.

- This source moves together with the $\mathrm{TB}$ in a conservative manner, supplying the necessary disconnections to keep the shear-coupled migration.

All these points can be analyzed a priory using the topological theory of interfacial defects together with the dichromatic pattern, as a useful tool.

To conclude, there is a necessary crystallographic condition associated to the existence of the appropriate topological defects of the grain/twin boundary for the migration to occur. This necessary condition is prior to the achievement of the necessary physical parameters, linked to the atomic interactions.

Acknowledgements. This work was supported by the Euratom research and training programme 2014-2018 under grant agreements: No 661913 (SOTERIA) and No 755039 (Project M4F). This work also contributes to the Joint Program on Nuclear Materials (JPNM) of the European Energy Research Alliance (EERA). The research was partially supported by the Spanish MINECO (FIS2015-69017-P).

\section{References}

1. R. C. Pond, D.S. Vlachavas. Christallography, Proc. The Royal Society of London, Series A: Mathematical and Physical Sciences. 386, 95 (1983). Crossref

2. R.C. Pond. In: Dislocations in Solids, vol. 8, (ed. by F. R. N. Nabarro). North-Holland, Amsterdam (1989) p. 1

3. J.P. Hirth, R. C. Pond, R. C. Hoagland, X.-Y. Liu, J. Wang. Progress in Mater Sci. 58, 749 (2013). Crossref

4. J.P. Hirth, R.C. Pond. Acta Mater. 44, 4749 (1996). Crossref

5. Y. Hu, V. Turlo, I. J. Beyerlein, S. Mahajan, E. J. Lavernia, J. M. Schoenung, T. J. Rupert. Acta Mater. 194, 437 (2020). Crossref

6. H. El Kadiri, C.D. Barrett, J. Wang, C.N. Tomé. Acta Mater. 85, 354 (2015). Crossref

7. M. A. Tschopp, K. N. Solanki, F. Gao, X. Sun, M. A. Khaleel, M. F. Horstemeyer. Phys. Rev. B. 85, 064108 (2012). Crossref

8. D. Terentyev, X. He, A. Serra, J. Kuriplach. Comput. Mater. Sci. 49, 419 (2010). Crossref

9. R. Hadian, B. Grabowski, M. W. Finnis, J. Neugebauer. Phys. Rev. Mater. 2, 043601 (2018). Crossref

10. A. Rajabzadeh, F. Mompiou, M. Legros, N. Combe. Phys. Rev. Letters. 110, 265507 (2013). Crossref

11. N. Combe, F. Mompiou, M. Legros. Phys. Rev. B. 93, 024109 (2016). Crossref

12. H. A. Khater, A. Serra, R. C. Pond, J. P. Hirth. Acta Mater. 60, 2007 (2012). Crossref

13. R. C. Pond, J. P. Hirth, A. Serra, D. J. Bacon. Mater. Res. Letter. 4, 185 (2016). $\underline{\text { Crossref }}$

14. J. Wang, J.P. Hirth, C. N. Tomé. Acta Mater. 57, 5521 (2009). Crossref

15. J. Han, S. L. Thomas, D. J. Srolovitz. Progress in Mater. Sci. 98, 386 (2018). Crossref

16. A. Serra, D. J. Bacon. Philos. Mag. 73, 333 (1996). Crossref

17. T. Braisaz, P. Ruterana, G. Nouet, A. Serra, Th. Karakostas, T. Kehagias. Philos. Mag. Letters. 74, 331 (1996). Crossref

18. J. Wang, I. J. Beyerlein, J. P. Hirth, Model. Simul. Mater. Sci. Eng. 20 (2), 024001 (2012). Crossref

19. J. Wang, I. J. Beyerlein, J. P. Hirth, C. N. Tomé. Acta Mater. 59, 3990 (2011). Crossref

20. F. Mompiou, D. Caillard, M. Legros. Acta Mater. 57, 2198 (2009). Crossref

21. A. Rajabzadeh, M. Legros, N. Combe, F. Mompiou, D. A. Molodov. Philos. Mag. 93, 1299 (2013). Crossref

22. M. Mrovec, C. Elsässer, P. Gumbsch. Philos. Mag. 89, 3179 (2009). ․ㅏossref

23. B. Jiang, A. Tu, H. Wang, H. Duan, S. He, H. Ye, K. Du. Acta Mater. 155, 56 (2018). Crossref

24. H. W. Paxton. Acta Metall. 1, 141 (1953). Crossref

25. J. W. Christian, S. Mahajan. Progress Mater. Sci. A. 39, 1 (1995). Crossref

26. M. Rajagopalan, M. A. Tschopp, K. N. Solanki. JOM. 66, 129 (2014). Crossref

27. R. C. Pond, W. Bollmann. Phil. Trans. Roy. Soc. A. 292, 449 (1979).

28. A. Serra, D. J. Bacon. Philos. Mag. 90, 845 (2010). Crossref

29. N. Kvashin, P.L. García-Müller, N. Anento, A. Serra. Phys. Rev. Mater. 4, 073604 (2020). $\underline{\text { Crossref }}$ 
30. H. A. Khater, A. Serra, R. C. Pond. Philos. Mag. 93, 1279 (2013). Crossref

31. A. Serra, D. J. Bacon. Z. Metallkd. 95, 242 (2004). Crossref

32. A. Serra, D. J. Bacon. Mater. Sci. Eng. A. 400, 496 (2005). Crossref

33. S. Mahajan, D.F. Williams. Int. Met. Rev. 18, 43 (1973). Crossref

34. N. Anento, A. Serra. Comp. Mater Sci. 179,109679 (2020). Crossref

35. H.A. Khater, G. Monnet, D. Terentyev, A. Serra. Int. J. Plast. 62, 34 (2014). Crossref

36. A. Serra, R.C. Pond, D. J. Bacon. Acta Metall. Mater. 39,1469 (1991). Crossref

37. H. A. Khater, D. J. Bacon. Acta Mater. 58, 2978 (2010). Crossref
38. K. D. Molodov, T. Al-Samman, D. A. Molodov, S. KorteKerzel. Acta Mat. 134, 267 (2017). $\underline{\text { Crossref }}$

39. A. Serra, D. J. Bacon, R.C. Pond. Acta mater. 47, 1425 (1999). Crossref

40. A. Ostapovets, R. Gröger. Model. Simul. Mater. Sci. Eng. 22, 025015 (2014). Crossref

41. B. Xu, L. Capolungo, D. Rodney. Scripta Mater. 68, 901 (2013). Crossref

42. A. Ostapovets, J. Buršík, R. Gröger. Philos. Mag. 95, 4106 (2015). Crossref

43. C.D. Barrett, H. El Kadiri. Acta Mater. 63, 1 (2014). Crossref

44. A. Ostapovets, A. Serra. Philos. Mag. 94, 2827 (2014). Crossref

45. R. C. Pond, A. Serra, D. J. Bacon. Acta Mater. 47, 1441 (1999). Crossref 